\title{
Memory effects in polyethylenes: influence of processing and crystallization history
}

\author{
C. W. M. Bastiaansen* \\ DSM Research, PO Box 18,6160 MD Geleen, The Netherlands
}

H. E. H. Meyer

DSM Research, PO Box 18,6160 MD Geleen, The Netherlands and

Eindhoven University of Technology, Department of Polymer Technology, Eindhoven, The Netherlands

and $P$. J. Lemstra

Eindhoven University of Technology, Department of Polymer Technology, Eindhoven, The Netherlands

(Received 19 June 1989; revised 14 November 1989; accepted 17 November 1989)

\begin{abstract}
The experimental timescale of memory effects related to restoring equilibrium viscoelastic properties in solution-cast linear and long-chain branched polyethylenes has been investigated. It was found that the crystallization history of low-density polyethylene and ultra-high-molecular-weight polyethylene, with respect to solid-state and melt properties, is lost after heating into the melt after a short period of time. The experimental timescale of memory effects is at least one order of magnitude shorter than the longest relaxation times in these materials, which correspond to reptative motion of macromolecules. The experimental results indicate that long-term memory effects related to the physical entanglement network in polyethylenes are absent and that equilibrium viscoelastic properties of polyethylene melts and solids and the concept of entanglement coupling are related to segmental rather than to macromolecular mobility.
\end{abstract}

(Keywords: entanglement coupling; memory; polyethylene; crystallization; shear refining; dissolution)

\section{INTRODUCTION}

Polymeric materials form a unique class of materials with respect to pronounced viscoelastic phenomena, which are related to their long-chain character/nature. In the interpretation of viscoelastic behaviour, entanglement coupling is often invoked. To demonstrate the widespread use of the concept of entanglement coupling, we briefly recapitulate some experimental studies related to polymer melts, solutions and solids.

Extensive studies have been performed concerning the phenomenon of entanglement coupling in polymer melts. The plateau modulus of polymer melts is related to the entanglement network ${ }^{1,2}$, and the apparent molecular weight between entanglements $\left(M_{\mathrm{e}}\right)$ is deduced for monodisperse systems. Also the onset of the 3.4 power dependence of the zero-shear melt viscosity on the molecular weight is related to the onset of entanglement coupling, and $M_{\mathrm{e}}$ values are derived ${ }^{2-4}$.

In equilibrium melts, the value of $M_{\mathrm{e}}$ is independent of the molecular weight of the sample ${ }^{1-4}$. However, attempts have been made to modify the properties of polymer melts, for example by shear refining ${ }^{5-13}$. Upon repeated extrusion the melt viscosity could be lowered in the case of low-density polyethylene (LDPE), which was related to a decrease in entanglement density. Prolonged heating in the molten state and specific dissolution treatments restored the equilibrium properties, demonstrating that the process of shear refining is of a physical rather than a chemical nature.

* To whom correspondence should be addressed
Upon dissolution, the entanglement density can be reduced drastically. The increase in apparent molecular weight between entanglements is about inversely proportional to the polymer volume fraction until the dilute regime is reached. In the dilute regime, below the polymer overlap concentration, entanglement coupling is absent ${ }^{1,2}$.

Solvents have been used not only to improve processability of polymeric materials but also to induce a strongly enhanced drawability in the solid state. In contrast to melt-crystallized ultra-high-molecular-weight polyethylene (UHMW-PE), solution-spun/cast UHMW$\mathrm{PE}$ can be drawn in the solid state to high draw ratios even after complete removal of solvent from the films or fibres ${ }^{14-18}$. The experimentally observed dependence of the maximum attainable draw ratio on the polymer concentration in solution was explained semiquantitatively in terms of reduction of trapped entanglements, which act as physical crosslinks upon drawing.

In the past, several attempts have been made to visualize entanglements in polymers ${ }^{1,2}$. In Figure $l$ a rather familiar picture of an entanglement, four strands leading away from a contact, is shown. In this case a change in entanglement density in polymers is only possible via diffusion involving macromolecular reptative motion since chains cannot cross mutually.

Further information with respect to the physical nature of entanglement coupling can potentially be obtained from experimental studies on the timescale of memory effects in polymers with a reduced entanglement density. For instance, if the assumed physical nature of 


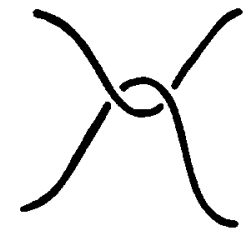

Figure 1 Schematic drawing of an entanglement

entanglements in Figure 1 is correct, long-term memory effects are expected in 'disentangled' polymers because 're-entangling' invokes reptation of macromolecules, which is a time-consuming process ${ }^{19-21}$.

Unfortunately, experimental studies on memory effects in disentangled polymers are of a contradictory nature. Both long-term ${ }^{5-13,22}$ and short-term memory effects ${ }^{23-25}$ have been reported. In this study detailed experimental results on memory effects in LDPE and UHMW-PE are presented. Special attention is devoted to the lifetime of memory effects in relation to macromolecular mobility and the origin of entanglement coupling. LDPE and UHMW-PE are chosen as model materials because of their long relaxation times in the melt. Consequently, long-term memory effects can potentially be observed in these materials.

\section{EXPERIMENTAL}

The polyethylene grades used in this study are Stamylan 2800 (LDPE, $M_{\mathrm{w}}=1.5 \times 10^{2} \mathrm{~kg} \mathrm{~mol}^{-1}$ ) and Hifax 1900 (UHMW-PE, $M_{\mathrm{w}}=5 \times 10^{3} \mathrm{~kg} \mathrm{~mol}^{-1}$ ).

Polyethylene solutions in xylene with a nominal polymer concentration of $1 \% \mathrm{w} / \mathrm{v}$ were prepared at $130^{\circ} \mathrm{C}^{15}$. Prior to the dissolution procedure, $1 \% \mathrm{w} / \mathrm{v}$ dibutyl-p-cresol was added to the polymers to prevent degradative oxidation. After complete dissolution had occurred, which took approximately $1 \mathrm{~h}$, the solutions were cast/quenched to room temperature. Subsequently, the solvent was evaporated at ambient conditions. In the case of LDPE, brittle, incoherent and porous films were obtained. These films were compression moulded in the solid state at $75^{\circ} \mathrm{C}$.

Solution-cast films, wrapped in aluminium foil, were melted/recrystallized by immersing them in a preheated silicone oil bath. After a preset time the films were quenched to room temperature.

Melt-crystallized LDPE and UHMW-PE films were prepared by compression moulding of respectively pellets and reactor powders at $180^{\circ} \mathrm{C}$ for $30 \mathrm{~min}$ at a pressure of $10 \mathrm{MPa}$. It is assumed that these melt-crystallized LDPE and UHMW-PE samples possess equilibrium viscoelastic properties in the solid state and melt.

In order to investigate memory effects in shear-refined polyethylenes, LDPE samples were repeatedly extruded in a co-rotating twin-screw extruder. The residence time in the extruder was approximately $2 \mathrm{~min}$ and the maximum temperature was $210^{\circ} \mathrm{C}$. To prevent thermal degradation of the LDPE, $0.5 \mathrm{wt} \%$ Irganox $^{(m)} 1044$ and $0.1 \%$ 2,5-di-t-butyl-4-methylphenol were added to the polymer. After each extrusion a sample was collected for measurements and the LDPE was re-extruded. In this way LDPE samples were produced that had passed through the extruder 1-5 times. Melt-crystallized films were prepared from shear-refined LDPE samples by compression moulding at $160^{\circ} \mathrm{C}$ for $15 \mathrm{~min}$.

Stress-strain measurements in the solid state were performed on a Zwick Tensile Tester equipped with a thermostatically controlled oven. Dumbbell-shaped samples with an original length of $10 \mathrm{~mm}$ were drawn at a constant crosshead speed of $100 \mathrm{~mm} \mathrm{~min}^{-1}$.

Dynamic viscosity measurements and step strainstress relaxation measurements were performed on a Rheometrics Mechanical Spectrometer, type RMS 7200, equipped with a parallel-plate system. Before the measurements were started, the samples were heated for approximately $3 \mathrm{~min}$.

Uniaxial drawing experiments in the melt were performed on a Göttfert Rheostrain at a constant strain rate of $0.1 \mathrm{~s}^{-1}$. Dumbbell-shaped samples with an original length of $20 \mathrm{~mm}$ were used. Before the measurements, the samples were heated for approximately $1 \mathrm{~min}$.

\section{RESULTS}

The lifetime of memory effects related to restoring equilibrium viscoelastic properties in repeatedly extruded and solution-cast polyethylenes was investigated. In general it is assumed that dissolution treatments and processing treatments have a similar effect on polyethylenes, i.e. a disentangled polymer is obtained ${ }^{5-18}$. The timescale of memory effects in repeatedly extruded 'shear-refined' LDPE is investigated and compared with the timescale of memory effects in solution-cast LDPE and UHMW-PE. Relaxation-time spectra in the melt are also measured and related to the lifetime of memory effects and macromolecular mobility.

\section{Repeatedly extruded 'shear-refined' LDPE}

LDPE was extruded five times at $220^{\circ} \mathrm{C}$ using a co-rotating twin-screw extruder. After each run a sample was collected for measurements.

In Figure 2 elongational flow measurements in the melt on repeatedly extruded LDPE samples are shown. The LDPE samples were subjected to a constant strain rate of $0.1 \mathrm{~s}^{-1}$ and the viscosity was measured as a function of time. The Hencky strain of the LDPE samples is

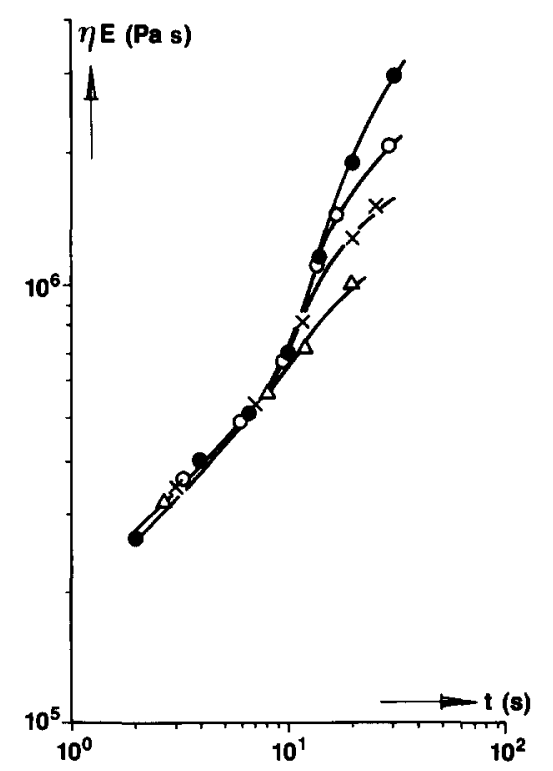

Figure 2 Elongational flow viscosity, at a strain rate of $0.1 \mathrm{~s}^{-1}$ and a temperature of $120^{\circ} \mathrm{C}$, as a function of time $t:(\bullet)$ as received; $(0)$ once extruded; $(x)$ three times extruded; $(\Delta)$ five times extruded 


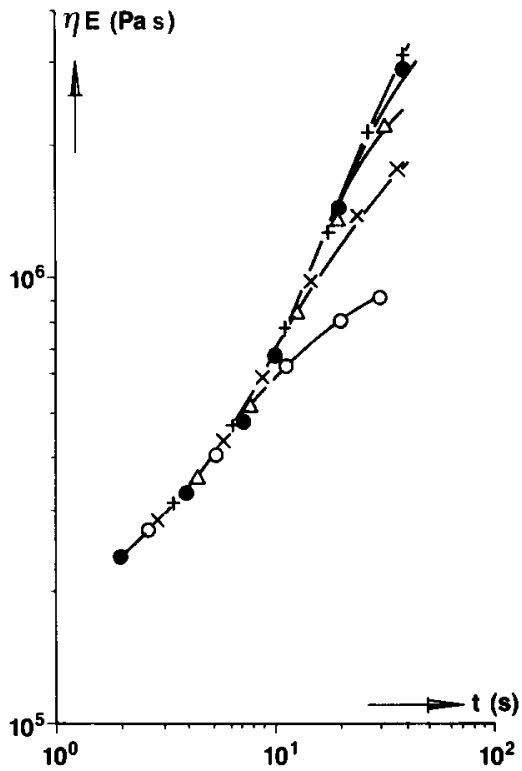

Figure 3 Elongational flow viscosity, at a strain rate of $0.1 \mathrm{~s}^{-1}$ and a temperature of $120^{\circ} \mathrm{C}$, as a function of time $t:(0)$ as received; $(O)$ five times extruded; $(x)$ five times extruded, followed by $2 \mathrm{~h}$ at $160^{\circ} \mathrm{C}$; $(\triangle)$ five times extruded, followed by $2 \mathrm{~h}$ at $200^{\circ} \mathrm{C} ;(+)$ five times extruded, cast from solution

equivalent to the strain rate multiplied by time. A substantial drop in elongational viscosity is observed especially at large Hencky strains. In Figure 3 it is shown that the observed drop in elongational viscosity can be reversed by annealing at high temperatures and has a lifetime of the order of $2 \mathrm{~h}$ at $200^{\circ} \mathrm{C}$. The drop in elongational viscosity of LDPE upon shear refining can also be reversed using specific dissolution treatments (Figure 3). The repeatedly extruded LDPE samples are dissolved in xylene at $130^{\circ} \mathrm{C}$. Subsequently the solutions are quenched to room temperature and the solvent is evaporated at ambient conditions. In general, the drop in elongational viscosity of LDPE upon shear refining is attributed to a reduction in entanglement density ${ }^{5-13}$. The experimental data therefore seem to indicate that a disentangled state in LDPE is preserved upon heating into the melt after a prolonged period of time.

\section{Solution-cast LDPE}

In Figure 4 nominal stress-strain curves, recorded in the solid state, of solution-cast (S) and melt-crystallized (M) LDPE are shown. The solution-cast sample was crystallized from a solution with a polymer concentration below the critical concentration for coil overlap. Consequently the connectivity between individual crystals after quenching to room temperature is completely lost, which results in a low maximum attainable draw ratio (Figure 4). An identical situation is obtained in the case of UHMW-PE but then at a much lower concentration ${ }^{15}$. The solid-state drawing behaviour of solution-cast LDPE after heating in the melt for $60 \mathrm{~s}$ $\left(\mathrm{S}^{*}\right)$, followed by a recrystallization, is virtually indistinguishable from the melt-crystallized reference sample $(\mathbf{M})$.

In Figures 5 and 6 dynamic mechanical measurements and elongational flow measurements of solution-cast (S) and melt-crystallized (M) LDPE samples are shown. The dynamic mechanical and elongational flow measurements were started after heating for respectively $3 \mathrm{~min}$ and $1 \mathrm{~min}$ into the melt to allow for thermal equilibrium. In both cases no differences were observed in rheological behaviour between the solution-cast (S) and meltcrystallized (M) samples.

In Figure 7 dynamic mechanical measurements of melt-crystallized LDPE are shown. The phase angle and

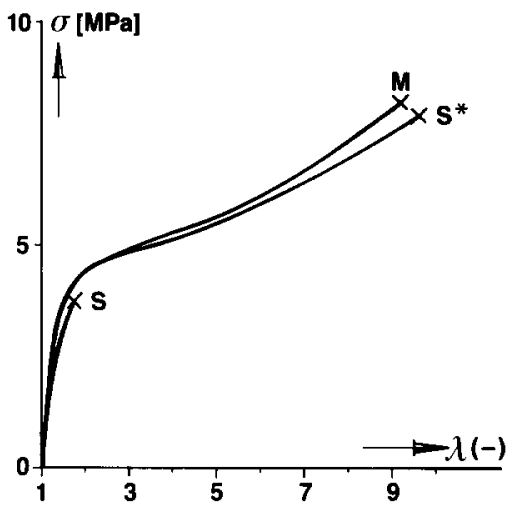

Figure 4 Nominal stress-strain curves of LDPE at $75^{\circ} \mathrm{C}$ : $\mathrm{M}$, melt-crystallized LDPE; S, solution-cast LDPE; $\mathrm{S}^{*}$, solution-cast, recrystallized LDPE $\left(1 \mathrm{~min}\right.$ at $\left.140^{\circ} \mathrm{C}\right)$

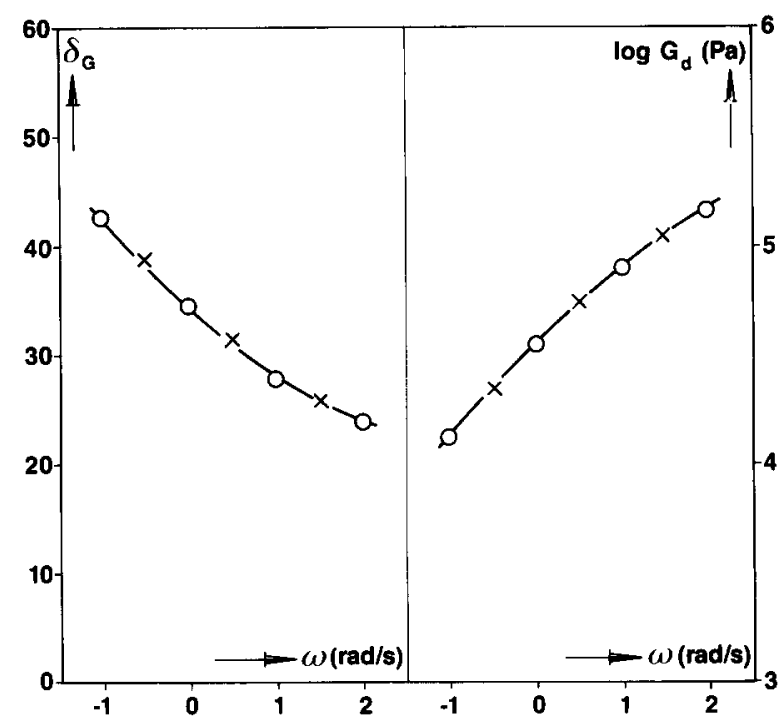

Figure 5 Complex dynamic modulus $G_{\mathrm{d}}$ and dynamic loss factor $\delta_{\mathrm{G}}$ at $120^{\circ} \mathrm{C}$ of melt-crystallized $(x)$ and solution-cast $(O)$ LDPE

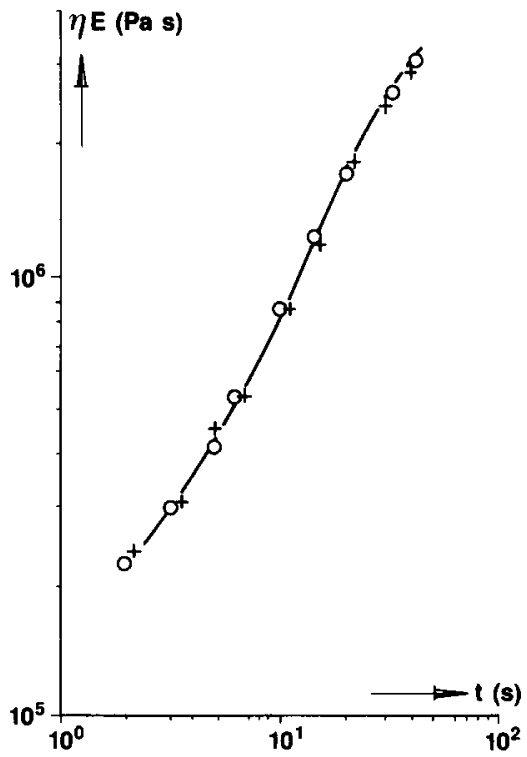

Figure 6 Elongational flow viscosity, at a strain rate of $0.1 \mathrm{~s}^{-1}$ and a temperature of $120^{\circ} \mathrm{C}$, as a function of time $t:(+)$ melt-crystallized LDPE; (O) solution-cast LDPE 


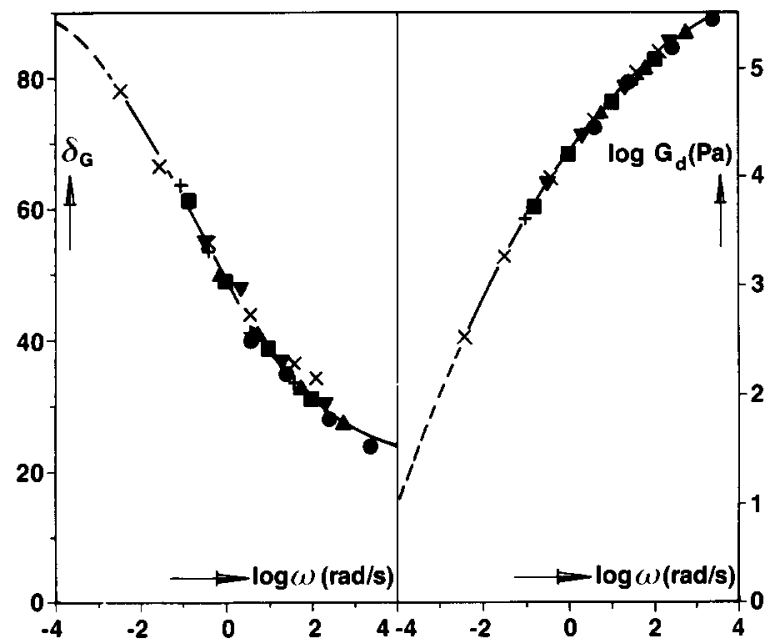

Figure 7 Master curve at $190^{\circ} \mathrm{C}$ of the phase angle and dynamic modulus of LDPE

dynamic modulus are measured in a temperature range from 120 to $210^{\circ} \mathrm{C}$ for angular frequencies ranging from $10^{-1}$ to $10^{2} \mathrm{rad} \mathrm{s}^{-1}$. A master curve was constructed by first shifting the phase angle along the horizontal axis, then shifting the modulus curves over the same distance along the frequency axis and finally effecting superposition of the modulus curves via a small shift along the modulus axis $^{26}$. From this master curve the relaxation-time spectrum can be derived. The frequency at which the phase angle starts to deviate from $90^{\circ}$ is of the order of $10^{-4} \mathrm{rad} \mathrm{s}^{-1}$ at $190^{\circ} \mathrm{C}$. This deviation occurs when the product of the angular frequency and longest relaxation times in the material is approximately unity. In other words, the experimental results in Figure 7 indicate that relaxation times up to $10^{4} \mathrm{~s}$ are present in LDPE melts at $190^{\circ} \mathrm{C}$. The longest relaxation times in LDPE compare well with the experimental timescale of memory effects in shear-refined LDPE. On the other hand, these relaxation times are at least one order of magnitude longer than the experimental timescale of memory effects in solution-cast LDPE.

\section{Ultra-high-molecular-weight linear polyethylene}

Experimental results on memory effects in solutioncast UHMW-PE have already been reported previously ${ }^{23,24}$. However, in those studies a relatively low-molecular-weight sample $\left(M_{\mathrm{w}} \sim 1.5 \times 10^{3} \mathrm{~kg} \mathrm{~mol}^{-1}\right)$ with a high polydispersity $\left(Q=M_{\mathrm{w}} / M_{\mathrm{n}}=10-15\right)$ was used, which causes problems in interpreting the experimental data ${ }^{23}$. Therefore the experiments performed in ref. 23 were repeated using a UHMW-PE grade with a higher weight-average molecular weight $\left(M_{\mathrm{w}}=5 \times 10^{3}\right.$ $\left.\mathrm{kg} \mathrm{mol}^{-1}\right)$ and a lower polydispersity $(Q=3-5)$.

Stress-strain measurements in the solid state and dynamic mechanical measurements in the melt of this particular UHMW-PE grade are shown in Figures 8 and 9 , respectively. In the dynamic mechanical measurements $3 \mathrm{~min}$ were allowed to reach thermal equilibrium. Again it is observed that the dissolution history of UHMW-PE with respect to solid-state and melt properties is lost almost instantaneously upon heating into the melt (Figures 8 and 9). Unfortunately, uniaxial drawing experiments in the true melt $\left(T>160^{\circ} \mathrm{C}\right)$ cannot be performed in the case of UHMW-PE samples ${ }^{25,27}$ because the samples fail at small uniaxial deformations. Melt drawing experiments can be performed in a temperature region from 140 to $155^{\circ} \mathrm{C}^{25,27}$. However, strain-induced crystallization takes place upon drawing in this temperature region, which makes the experimental results difficult to interpret.

In Figure 10 stress relaxation measurements of melt-crystallized UHMW-PE are shown. The measurements were stopped after approximately $3 \mathrm{~h}$. This figure shows that a large portion of long relaxation times $\left(\gg 10^{3} \mathrm{~s}\right)$ is present in this particular UHMW-PE grade.

The experimental results on memory effects in solution-cast UHMW-PE resemble those obtained with solution-cast LDPE. The dissolution history of solutioncast UHMW-PE is lost almost instantaneously upon heating in the melt and at timescales that are orders of magnitude shorter than the longest relaxation times.

\section{DISCUSSION}

Crystallization from dilute solution, especially if crystallization occurs from solutions below the overlap concentration, is an effective way to obtain disentangled polymer systems. The properties of solution-cast LDPE and UHMW-PE, as presented in Figures 4 and 8, can be easily explained in terms of strongly reduced entanglement density. The disentangled situation in solution is preserved in the solid as a consequence of crystallization into chain-folded crystals.

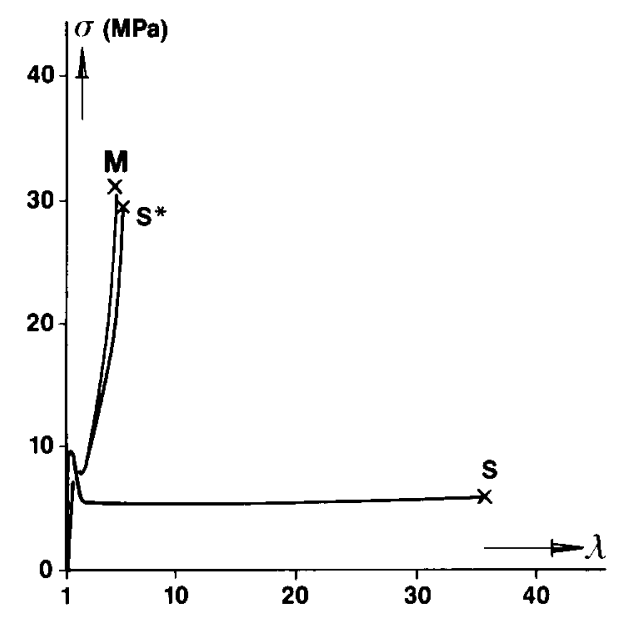

Figure 8 Nominal stress-strain curves of UHMW-PE at $90^{\circ} \mathrm{C}$ : $\mathrm{M}$, melt-crystallized UHMW-PE; S, solution-cast UHMW-PE; $\mathrm{S}^{*}$, solution-cast, recrystallized UHMW-PE $\left(1 \mathrm{~min}\right.$ at $\left.150^{\circ} \mathrm{C}\right)$

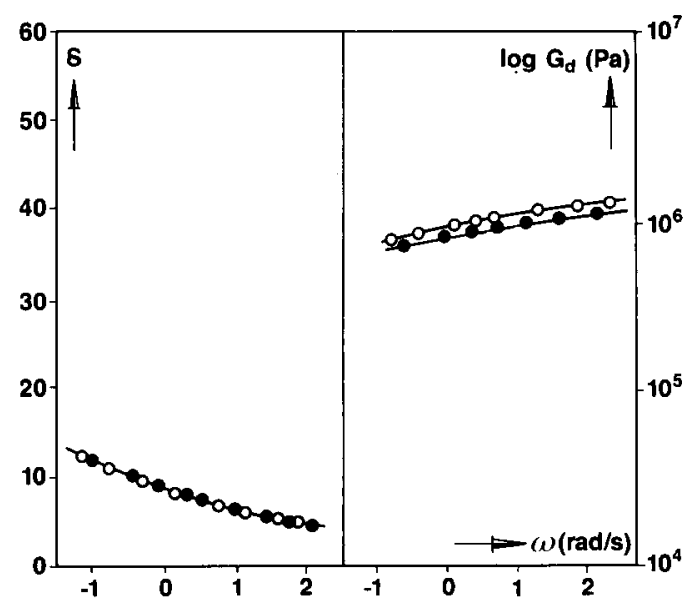

Figure 9 Complex dynamic modulus $G_{\mathrm{d}}$ and dynamic loss factor $\delta$ at $160^{\circ} \mathrm{C}$ of melt-crystallized $(O)$ and solution-cast (e) UHMW-PE 


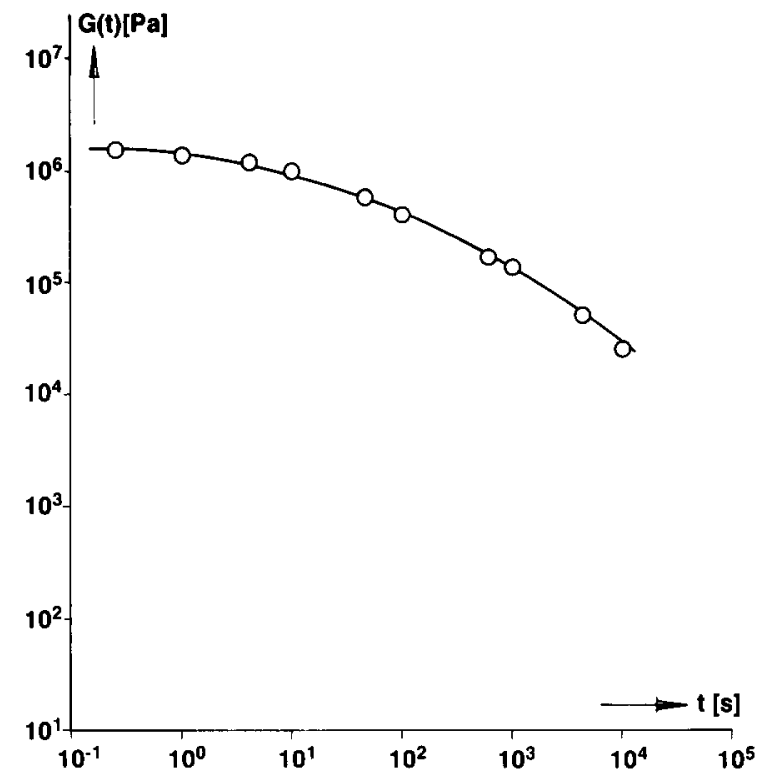

Figure 10 Stress relaxation measurements of melt-crystallized UHMW-PE at $180^{\circ} \mathrm{C}$

If we adopt the classical representation of an entanglement, as presented in Figure 1, no difficulties are encountered in understanding the process of disentangling via dissolution. Chains as a whole are separated upon dissolution, and at polymer concentrations below the critical concentration for coil overlap isolated chains are obtained and the number of intermolecular contacts is reduced to the extreme. The surprising observation, however, is the short timescale needed to destroy the solution-induced drawing characteristics.

In the solid state the disentangled status can be preserved indefinitely whereas heating above the melting point for a short time renders a solid (after recrystallization) or a melt that are indistinguishable from their equilibrium counterpart (Figures 4-6, 8 and 9). In other words no time effects could be measured for restoring equilibrium properties.

Upon melting of solution-crystallized chain-folded PE crystals, the chains will expand towards a random-coil conformation $^{28}$. The driving force will be of an entropic nature. In order to restore an equilibrium entanglement density, the formation of entanglements, as schematically represented in Figure 1 , is virtually impossible since reptative motion of complete chains is needed at corresponding timescales beyond the experimental times used in this study. The experimental results therefore indicate that equilibrium properties of polyethylene melts and the concept of entanglement coupling are related to segmental rather than to macromolecular mobility.

The experimental results suggest that local segmental reorganization is sufficient to destroy the solutioninduced, disentangled state in both the melt and the solid state. Local reorganization of chain segments upon heating in the melt results in less adjacent re-entry in the crystals upon recrystallization ${ }^{29}$. It has already been suggested that such a local reorganization of stems within crystals, as a result of recrystallization, might hamper solid-state deformation ${ }^{27}$, which can explain the observed fast decay in ultra-durability of UHMW-PE upon recrystallization from a kinetic viewpoint.

Seemingly in contrast, however, the shear-refined LDPE samples show pronounced memory effects. In a first approximation the timescale of the observed memory effects corresponds to the longest relaxation times in LDPE (Figures 3 and 7). In view of the results concerning solution-crystallized LDPE, these memory effects cannot be simply attributed to restoring an equilibrium entanglement network from a disentangled situation. In our opinion, shear refining is less effective compared to crystallization from dilute solution to obtain a disentangled situation. Alternative explanations have been suggested in the literature, for example by Münstedt, involving alignment of long-chain branches of LDPE along the macromolecular backbone upon shear refining ${ }^{9}$. This concept is supported by the experimentally observed absence of an upswing in elongational viscosity at large Hencky strains in repeatedly extruded LDPE, which is typically a property of linear polyethylenes ${ }^{30}$.

\section{CONCLUSIONS}

The dissolution history of linear and long-chain branched polyethylenes with respect to solid-state and melt properties is lost almost instantaneously upon heating into the melt. In other words, long-term memory effects related to the disentangled state in solution-cast polyethylenes are not observed. These experimental results indicate that reptation of chains as a whole is not a prerequisite to restore equilibrium properties in polyethylenes and that viscoelastic properties in the solid state and melt are related to segmental rather than to macromolecular mobility. A consequence of this experimental observation is that entanglements of the type shown in Figure 1 have a minor influence on the viscoelastic properties of linear and long-chain branched polyethylenes in the solid state and melt.

Memory effects with a physical origin and a long lifetime are observed in repeatedly extruded LDPE. The experimental results on solution-cast LDPE indicate that the observed phenomena cannot be simply attributed to modification of the entanglement network but are probably caused by an alignment of long-chain branches along the macromolecular backbone or a related intramolecular chain topology.

\section{ACKNOWLEDGEMENTS}

The authors wish to thank $\mathrm{Mr}$ J. H. M. Palmen and $\mathrm{Mr}$ T. Sleypen (DSM Research) for performing the rheological measurements and Dr P. J. R. Leblans (DSM Research) for many stimulating discussions.

\section{REFERENCES}

1 Ferry, J. D. 'Viscoelastic Properties of Polymers', 2nd Edn, Wiley, New York, 1970

2 Graessley, W. W. Adv. Polym. Sci. 1974, 16, Ch. 7

3 Berry, G. C. and Fox, T. G. Adv. Polym. Sci. 1968, 5, 261

4 Allen, V. R. and Fox, T. G. J. Chem. Phys. 1964, 41, 337

5 Rokudai, M. J. Appl. Polym. Sci. 1979, 23, 436

6 Rokudai, M., Mihara, S. and Fujiki, T. J. Appl. Polym. Sci. 1979, 26, 3289

7 Rokudai, M. and Fujiki, T. J. Appl. Polym. Sci. 1981, 26, 1343

8 Maxwell, B. and Breckwoldt, A. J. Rheol. 1981, 25, 55

9 Münstedt, H. Colloid. Polym. Sci. 1981, 259, 966

10 Teh, J. W., Rudin, A. and Schreiber, H. P. J. Appl. Polym. Sci. $1985,30,1345$

11 Ram, A. and Izrailov, L. J. Appl. Polym. Sci. 1986, 31, 85

12 Ritzau, G. Int. Polym. Proc. 1987, 1, 188

13 Schreiber, H. P. J. Polym. Eng. Sci. 1983, 23, 422 
14 Smith, P. and Lemstra, P. J. US Patents 4344908, 4422993, 4430383, 4436689 (DSM Stamicarbon)

15 Smith, P., Lemstra, P. J. and Booy, H. C. J. Polym. Sci., Polym. Phys. Edn 1981, 19, 877

16 Smith, P. and Lemstra, P. J. J. Mater. Sci. 1980, 15, 505

17 Smith P. and Lemstra, P. J. J. Polym. Sci., Polym. Phys. Edn $1981,19,1007$

18 Smith, P., Lemstra, P. J. and Pypers, J. P. L. J. Polym. Sci., Polym. Phys. Edn 1982, 20, 2229

19 De Gennes, P. G. 'Scaling Concepts in Polymer Physics', Cornell University Press, Ithaca, NY, 1978, Ch. 8

De Gennes, P. G. J. Chem. Phys. 1971, 55, 572

Klein, J. and Briscoe, B. J. Proc. R. Soc. Lond. (A) 1979, 365, 53

Rault, J. CRC Crit. Rev. Solid State Mater. Sci. 1986, 13, 57

Lemstra, P. J. and Kirschbaum, R. Polymer 1985, 26, 1372
Bastiaansen, C. W. M., Froehling, P., Pypers, A. J. and Lemstra, P. J. 'Integration of Polymer Science and Technology', Elsevier Applied Science, London, 1985, p. 508 Angew. Makromol. Chem. 1986, 145/146, 343

Scholtens, B. J. R. and Booy, H. C. 'Elastomers and Rubber Elasticity', ACS Symp. Ser. 139, American Chemical Society, Washington, DC, 1982, p. 517

27 Lemstra, P. J, Van Aerle, N. A. J. M. and Bastiaansen, C. W. M. Polymer $J .1987,19,85$

28 Sadler, D. M. and Keller, A. Science 1979, 203, 263

29 Keller, A. Faraday Disc. Chem. Soc. 1979, 68, 145

30 Leblans, J. R. and Bastiaansen, C. W. M. Macromolecules accepted 